\title{
On the solution of a problem of extended thermoelasticity theory (ETE) by using a complete finite element approach
}

\author{
O.N. Shivay*, S. Mukhopadhyay \\ Department of Mathematical Sciences \\ Indian Institute of Technology (BHU) \\ Varanasi-221005, India \\ *E-mail: onsj79@gmail.com
}

Received: 08 December 2018; revised: 04 May 2019; accepted: 06 May 2019; published online: 12 June 2019

\begin{abstract}
This paper attempts to apply a complete finite element approach for the solution of problems on coupled dynamical thermoelasticity theory. Presently, we employ the extended thermoelasticity theory proposed by Lord and Shulman (1969) and consider a problem of linear thermoelasticity for the hollow disk with a thermal shock applied on its inner boundary. The thermoelastic equations have been solved using the complete finite element approach, where we have used discretization in the time domain as well as space domain and applied the Galerkin's approach of the finite element for both time and space domain. We implement our scheme for a particular case and carry out computational work to obtain the numerical solution of the problem. Further, we compare the present results with the solutions obtained by FEM with Newmark time integration method and the solutions obtained by a trans-FEM method in which Laplace transform technique is used for the time domain. We show that, there is a perfect match in solutions of complete finite element approach with trans-finite element method and Newmark method. The efficiency of the method with respect to computation time is also compared with other two methods.
\end{abstract}

Key words: coupled thermoelasticity, extended thermoelasticity theory, finite element method, Newmark method, transFEM

\section{INTRODUCTION}

According to the classical uncoupled theory of heat conduction, the conduction of heat in a material is only dependent on the temperature gradient, and the theory ignores the effect of other mechanical causes, like the effect of elastic property of the material on the heat transfer. For the large time scale or small heat flux this theory behaves well, but for the short time scale or high heat flux values it fails to explain some physical phenomena completely. Also, the heat conduction equation based on the theory is of parabolic type in nature and hence predicts the infinite speed of heat transfer, which is not acceptable either from the physical or experi- mental view. Therefore, many researchers have tried to overcome such situations and proposed some modification to the classical theory.

Biot [1] introduced the coupling of thermal and elastic fields based on the Fourier's law of heat conduction. This theory was the first attempt to consider the mutual interaction of thermal and mechanical fields during thermo-mechanical loading on an elastic body. Hence Biot's theory takes into account the elastic effects on the heat conduction and vice versa. However, this theory suffers from the drawback of infinite speed of heat propagation due to the involvement of the classical heat conduction law in this theory. Chandrasekharaiah $[2,3]$ discussed the development of some mod- 
ified thermoelasticity theories predicting finite heat propagation speed and some investigations based on these modified models. Catteneo [4] and Vernotte [5, 6] suggested a modification to the classical theory and proposed a time delay parameter in the heat flux vector, which removes the drawback of infinite heat speed. Lord and Shulman [7] implemented this modification for the coupled thermoelasticity theory and introduced an extended thermoelasticity (ETE) theory. They obtained a solution for this coupled theory and compared them with the conventional thermoelasticity theory. Later on, Green and Lindsay [8] proposed the temperature-ratedependent thermoelasticity theory by introducing two relaxation parameters in the stress-strain relation and entropy equation, while Fourier's law remained the same. Here, it is further worth mentioning that Green and Naghdi $[9,11]$ suggested a new concept of thermoelasticity theory. Hetnarski and Ignaczak [12], Hetnarski and Eslami [13] have given a detailed discussion for GL, LS and GN models and also discussed some theorems based on these models.

Obtaining a closed form solution of the system of coupled thermoelastic equations are possible only for a few simple initial boundary value problems, and closed form solution cannot be obtained for many practical problems. Due to this reason, in order to reduce the complexity of the problem some simplified forms of basic equations are assumed by observations based on practical experiments. In the structural designs the use of these approximated forms of basic equations may prove good for simple structures, but for heavy-duty equipment it does not give satisfactory responses. Hence, the calculation must be performed on the basis of all governing equations of thermoelasticity theory in such cases. Usually, numerical methods can be used in these cases. Among all the numerical methods the finite element method can be applied efficiently to various coupled thermoelastic problems (see Prakash et al. [14], Mishra et al. [15] and $\mathrm{Xu}$ and $\mathrm{Li}$ [16]). Some authors have used the Galerkin's approach of the finite element along with the numerical transformation techniques (Laplace transform) for the time domain to solve different thermoelasticity problems. In this approach Laplace transformation is taken for the time domain, and then the finite element equation is derived for the space domain to get the solution in Laplace transformation form. Further, the suitable method for numerical inversion of the transformed solution gives the final solution of the coupled equations. A problem of the hollow disc for various thermoelasticity model using FEM with Laplace transformation has been studied by Bagri and Eslami [17], Kothari and Mukhopadhyay [18]. To solve coupled thermoelastic equations using the finite element method Rincon et al. [17] have discussed an alternative way to the transformation technique for time domain. They have suggested the discretization in space for deriving the finite element equation and use of implicit Newmark scheme to get the solution in the time domain. Abbas and Alzahrani [18] have used FEM with the implicit temporal integration method to solve a mode-I crack problem in two dimensional isotropic medium based on GN thermoelasticity theory. Stasa [19] has given a detailed discussion for FEM to various structural problems and suggested the complete finite element approach for space as well as time domain. Balla [20] has derived the explicit formulation of field variables for a two-dimensional heat conduction equation based on the $\mathrm{CV}$ model using this approach.

The main motive of this work is to apply the approach of solving coupled linear thermoelastic equations efficiently, using a complete finite element approach. At first, we discretize the space domain into small elements of equal intervals and use weak formulation to derive the finite element equation for the space domain. Next, we discretize the time domain, and again we apply the Galerkin's approach of finite element to obtain the explicit finite element equation for the time as well as space domain. We successfully implement this method and compute the solution of the problem for a hollow disk. A comparison of the solution to this problem with the solution obtained by FEM with Laplace transform technique and with the solution obtained by FEM with the Newmark time integration method is presented to show that the solutions match perfectly. The computational efficiency of the method is found to be much higher as compared to trans-FEM and similar to the Newmark method. We believe that this work will be helpful to solve any problem of coupled thermoelasticity by applying this complete finite element method.

\section{BASIC GOVERNING EQUATIONS}

The basic equations of the linear thermoelasticity theory for homogeneous and isotropic medium following the theory of Lord and Shulman [7] (ETE or LS theory) can be considered as follows:

The stress-displacement equation of motion in absence of any body force:

$$
\tau_{i j, j}=\rho \ddot{u}_{i} .
$$

Basic constitutive relations:

$$
\begin{gathered}
\tau_{i j}=2 \mu \varepsilon_{i j}+\lambda \varepsilon_{k k} \delta_{i j}-\beta \theta \delta_{i j}, \\
q_{i}+t_{q} \frac{\partial q_{i}}{\partial t}=-k \theta_{, i}, \\
\rho T_{0} s=\rho c_{E} \theta+\beta T_{0} \varepsilon_{k k} .
\end{gathered}
$$

Strain-displacement relation:

$$
\varepsilon_{i j}=\frac{\left(u_{i, j}+u_{j, i}\right)}{2} .
$$

The energy balance equation without heat source:

$$
q_{i, i}=-\rho T_{0} \dot{s} .
$$


Therefore, the heat conduction equation can be obtained from equations (3),(4) and (6) as

$$
\left(1+t_{q} \frac{\partial}{\partial t}\right)\left(\rho c_{E} \dot{\theta}+\beta T_{0} \dot{\varepsilon}_{k k}\right)=k \theta_{, i i} .
$$

In above equations (1-7), $\tau_{i j}, u_{i}, \varepsilon_{i j}, q_{i}$ are the components of stress tensor, displacement vector, strain tensor and heat flux vector, respectively. $\theta=T-T_{0}, T$ is the temperature and $T_{0}$ is the reference temperature. $\lambda, \mu$ are Lame's constant. $\beta=(3 \lambda+2 \mu) \alpha, \alpha$ is the coefficient of linear thermal expansion. $s$ is the entropy per unit mass. $k$ is the thermal conductivity. $t_{q}$ is the delay term in the heat flux vector. $\rho$ is the density of the material, and $c_{E}$ is the specific heat of the material at constant strain. The subscripted comma denotes the space derivative, and superposed dots are indicating the time derivative. $\delta_{i j}$ is the Kronecker delta. The subscripts $i, j$ vary from one to three.

\section{1. Problem formulation}

In the present work, we consider a problem of radially symmetric motion of a hollow disk with inner radius $a$ and outer radius $b$ under the linear thermoelasticity theory based on LS-model as described above. The material of the disc is assumed to be homogeneous and isotropic for this problem. So, the equations (7) can be written in the polar form as

$$
\begin{array}{r}
k\left(\frac{\partial^{2} \theta}{\partial r^{2}}+\frac{1}{r} \frac{\partial \theta}{\partial r}\right)-\left(1+t_{q} \frac{\partial}{\partial t}\right)\left[\rho c_{E} \frac{\partial \theta}{\partial t}+\right. \\
\left.+\beta T_{0} \frac{\partial}{\partial t}\left(\frac{\partial u}{\partial r}+\frac{u}{r}\right)\right]=0
\end{array}
$$

Further, from equations (1) and (2), we obtain the polar form of the equation of motion in terms of displacement and temperature as

$$
(\lambda+2 \mu)\left[\frac{\partial^{2} u}{\partial r^{2}}+\frac{1}{r} \frac{\partial u}{\partial r}-\frac{u}{r^{2}}\right]-\beta \frac{\partial \theta}{\partial r}-\rho \frac{\partial^{2} u}{\partial t^{2}}=0 .
$$

The radial and circumference stress components can also be obtained from equations (1) and (2) as

$$
\begin{gathered}
\tau_{r r}=2 \mu \frac{\partial u}{\partial r}+\lambda\left(\frac{\partial u}{\partial r}+\frac{u}{r}\right)-\beta \theta, \\
\tau_{\phi \phi}=2 \mu \frac{u}{r}+\lambda\left(\frac{\partial u}{\partial r}+\frac{u}{r}\right)-\beta \theta .
\end{gathered}
$$

For this problem, we are considering friction less inner surface and apply the thermal shock on the inner boundary of the disk. The rigid outer boundary is assumed to be insulated. We also consider the homogeneous initial conditions. Hence, we have

$$
\left.\begin{array}{l}
u(r, 0)=0, \theta(r, 0)=0, \text { at } a \leq r \leq b \\
\dot{u}(r, 0)=0, \dot{\theta}(r, 0)=0, \text { at } a \leq r \leq b
\end{array}\right\}
$$

$$
\left.\begin{array}{lll}
\theta(a, t)=1-e^{-1000 t} & \text { and } \tau_{r r}(a, t)=0, & \text { at } t>0 \\
\frac{\partial \theta(b, t)}{\partial r}=0, & \text { and } u(b, 0)=0, & \text { at } t>0
\end{array}\right\}
$$

Now, to make the equations in simplified non-dimensional forms, we assume the following dimensionless variables:

$$
\begin{aligned}
& r^{\prime}=c_{1} n_{0} r, \quad \theta^{\prime}=\frac{\theta}{T_{0}}, t^{\prime}=c_{1}^{2} n_{0} t, \\
& t_{q}^{\prime}=c_{1}^{2} n_{0} t_{q}, \quad\left(\tau_{r r}^{\prime}, \tau_{\phi \phi}^{\prime}\right)=\left(\frac{\tau_{r r}}{\beta T_{0}}, \frac{\tau_{\phi \phi}}{\beta T_{0}}\right), \\
& u^{\prime}=\frac{c_{1} n_{0}(\lambda+2 \mu)}{\beta T_{0}} u, \tau_{i j}^{\prime}=\frac{\tau_{i j}}{\beta T_{0}}, \\
& c_{1}^{2}=\frac{(\lambda+2 \mu)}{\rho}, n_{0}=\frac{\rho c_{E}}{k} .
\end{aligned}
$$

Using the above dimensionless variables and ignoring the prime notations for simplicity, the equations (8-11) can be expressed as

$$
\begin{gathered}
(\lambda+2 \mu)\left[\frac{\partial^{2} u}{\partial r^{2}}+\frac{1}{r} \frac{\partial u}{\partial r}-\frac{u}{r^{2}}\right]-(\lambda+2 \mu) \frac{\partial \theta}{\partial r}-\rho c_{1}^{2} \frac{\partial^{2} u}{\partial t^{2}}=0 \\
\left(\frac{\partial^{2} \theta}{\partial r^{2}}+\frac{1}{r} \frac{\partial \theta}{\partial r}\right)-\left(1+t_{q} \frac{\partial}{\partial t}\right) \frac{\partial}{\partial t}\left[\theta+\xi\left(\frac{\partial u}{\partial r}+\frac{u}{r}\right)\right]=0 \\
\tau_{r r}=\frac{2 \mu}{\lambda+2 \mu} \frac{\partial u}{\partial r}+\frac{\lambda}{\lambda+2 \mu}\left(\frac{\partial u}{\partial r}+\frac{u}{r}\right)-\theta \\
\tau_{\phi \phi}=\frac{2 \mu}{\lambda+2 \mu} \frac{u}{r}+\frac{\lambda}{\lambda+2 \mu}\left(\frac{\partial u}{\partial r}+\frac{u}{r}\right)-\theta
\end{gathered}
$$

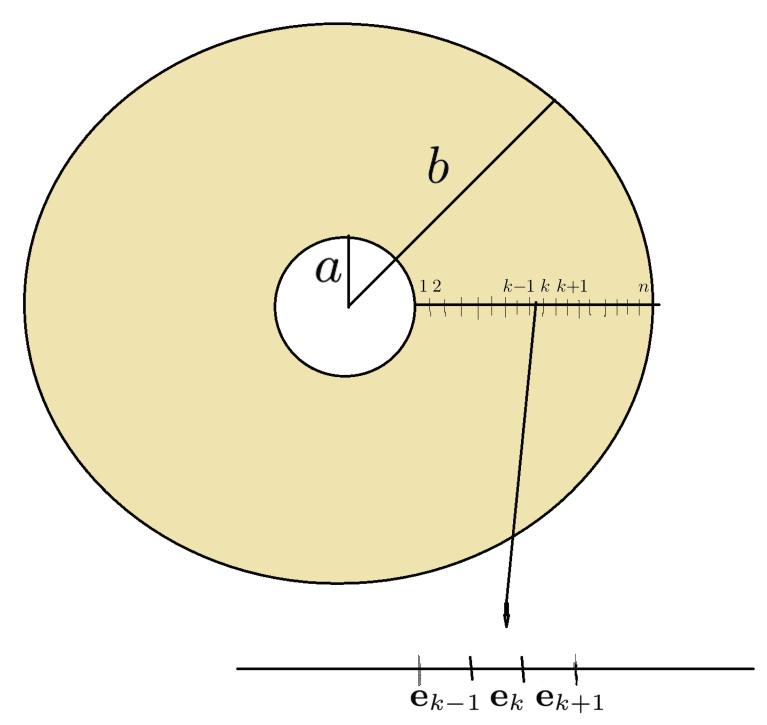

Fig. 1. Finite element profile of the Hollow disk 


\section{FINITE ELEMENT FORMULATION}

Now, to solve the coupled equations (14) and (15) with the initial conditions (12) and boundary conditions (13), we use the Galerkin approach of the finite element method. In this process, discretization of space domain into small subdomains takes place (see Fig. 1), and the temperature and displacement are approximated by the following functions for each sub-domain:

$$
u=\sum_{i=1}^{m} N_{i} U_{i}^{(e)}(t), \text { and } \theta=\sum_{i=1}^{m} N_{i} \Theta_{i}^{(e)}(t)
$$

where $N_{i}, i=1,2, \ldots, m$ are the shape functions and $U_{i}^{(e)}(t), \Theta_{i}^{(e)}(t), i=1,2, \ldots, m$ are the approximated nodal displacement and temperature, respectively.

Therefore, by applying Galerkin's approach and assuming the shape function as weight function with the approximation (18), the equations (14) and (15) can be written in the form

$$
\begin{gathered}
\int_{V} N_{i}\left[(\lambda+2 \mu)\left[\frac{\partial^{2} u}{\partial r^{2}}+\frac{1}{r} \frac{\partial u}{\partial r}-\frac{u}{r^{2}}\right]+\right. \\
\left.-(\lambda+2 \mu) \frac{\partial \theta}{\partial r}-\rho c_{1}^{2} \frac{\partial^{2} u}{\partial t^{2}}\right] d V=0, \\
\int_{V} N_{i}\left[\left(\frac{\partial^{2} \theta}{\partial r^{2}}+\frac{1}{r} \frac{\partial \theta}{\partial r}\right)+\right. \\
\left.-\left(1+t_{q} \frac{\partial}{\partial t}\right) \frac{\partial}{\partial t}\left[\theta+\xi\left(\frac{\partial u}{\partial r}+\frac{u}{r}\right)\right]\right] d V=0 .
\end{gathered}
$$

For a disk the infinitesimal volume element will be $r d r d \phi$, but in the present case of radial symmetry, all the variables are independent from the angle. Therefore, by considering the infinitesimal volume element as $d V=r d r$ and $r=r_{k}+\eta, k=1,2, \ldots, n$ in the equations (19) and (20), we have

$$
\begin{gathered}
\int_{0}^{h} N_{i}\left[(\lambda+2 \mu)\left[\frac{\partial^{2} u}{\partial \eta^{2}}+\frac{1}{\left(\eta+r_{i}\right)} \frac{\partial u}{\partial \eta}-\frac{u}{\left(\eta+r_{i}\right)^{2}}\right]+\right. \\
\left.-(\lambda+2 \mu) \frac{\partial \theta}{\partial \eta}-\rho c_{1}^{2} \frac{\partial^{2} u}{\partial t^{2}}\right]\left(\eta+r_{i}\right) d \eta=0
\end{gathered}
$$

and

$$
\begin{aligned}
& \int_{0}^{h} N_{i}\left\{\left[\frac{\partial^{2} \theta}{\partial \eta^{2}}+\frac{1}{\left(\eta+r_{k}\right)} \frac{\partial \theta}{\partial \eta}\right]-\left[1+t_{q} \frac{\partial}{\partial t}\right] \times\right. \\
& \left.\times\left[\frac{\partial \theta}{\partial t}-\xi \frac{\partial}{\partial t}\left(\frac{\partial u}{\partial \eta}+\frac{u}{\left(\eta+r_{k}\right)}\right)\right]\right\}\left(\eta+r_{k}\right) d \eta=0 .
\end{aligned}
$$

Now, applying integration by part in the first term of the (21) to derive the weak formulation, we get

$$
\begin{aligned}
& \left.(\lambda+2 \mu) \frac{\partial u}{\partial \eta}\left(\eta+r_{k}\right) N_{i}\right|_{0} ^{h}+ \\
& -\int_{0}^{h}\left[N_{i}(\lambda+2 \mu) \frac{\partial u}{\partial \eta}+(\lambda+2 \mu)\left(\eta+r_{k}\right) \frac{\partial N_{i}}{\partial \eta} \frac{\partial u}{\partial \eta}\right] d \eta+ \\
& +\int_{0}^{h}\left[N_{i}(\lambda+2 \mu)\left(\eta+r_{k}\right) \frac{\partial u}{\partial \eta}-N_{i} \frac{u}{\left(\eta+r_{k}\right)}\right] d \eta+ \\
& \quad+\int_{0}^{h} N_{i}\left[(\lambda+2 \mu) \frac{\partial \theta}{\partial \eta}+\rho c_{1}^{2} \frac{\partial^{2} u}{\partial t^{2}}\right]\left(\eta+r_{k}\right) d \eta=0,
\end{aligned}
$$

which implies that

$$
\begin{gathered}
\int_{0}^{h}\left[(\lambda+2 \mu)\left(\eta+r_{k}\right) \frac{\partial N_{i}}{\partial \eta} \frac{\partial u}{\partial \eta}+(\lambda+2 \mu) N_{i} \frac{u}{\left(\eta+r_{k}\right)}+\right. \\
\left.+\rho c_{1}^{2} N_{i} \frac{\partial^{2} u}{\partial t^{2}}\right] d \eta+\int_{0}^{h} N_{i}(\lambda+2 \mu) \frac{\partial \theta}{\partial \eta}\left(\eta+r_{k}\right) d \eta= \\
=\left.(\lambda+2 \mu)\left(\eta+r_{k}\right) N_{i}\right|_{0} ^{h} .
\end{gathered}
$$

Similarly, from equation (22), we have

$$
\begin{aligned}
& \left.N_{i} \frac{\partial \theta}{\partial \eta}\left(\eta+r_{k}\right)\right|_{0} ^{h}-\int_{0}^{h} \frac{\partial N_{i}}{\partial \eta} \frac{\partial \theta}{\partial \eta}\left(\eta+r_{k}\right) d \eta+ \\
& -\int_{0}^{h} N_{i}\left(\eta+r_{k}\right)\left(1+t_{q} \frac{\partial}{\partial t}\right) \frac{\partial \theta}{\partial t} d \eta+ \\
& -\int_{0}^{h} \xi\left(1+t_{q} \frac{\partial}{\partial t}\right) \frac{\partial}{\partial t}\left(\frac{\partial u}{\partial r}+\frac{u}{r}\right) N_{i} d \eta=0,
\end{aligned}
$$

i.e.

$$
\begin{aligned}
& \int_{0}^{h} \frac{\partial N_{i}}{\partial \eta} \frac{\partial \theta}{\partial \eta}\left(\eta+r_{k}\right) d \eta+ \\
& +\int_{0}^{h} N_{i}\left(\eta+r_{k}\right)\left(1+t_{q} \frac{\partial}{\partial t}\right) \frac{\partial \theta}{\partial t} d \eta+ \\
& +\int_{0}^{h} \xi\left(1+t_{q} \frac{\partial}{\partial t}\right) \frac{\partial}{\partial t}\left(\frac{\partial u}{\partial r}+\frac{u}{r}\right) N_{i} d \eta=0 .
\end{aligned}
$$

Now, using the equation (18) in equations (24) and (26) and taking $i=1,2$ we obtain the finite element equation formulation as 


$$
\begin{gathered}
U_{1}^{(e)}\left[(\lambda+2 \mu) \int_{0}^{h}\left[\left(\eta+r_{k}\right) \frac{\partial N_{i}}{\partial \eta} \frac{\partial N_{1}}{\partial \eta}+\frac{N_{i} N_{1}}{\left(\eta+r_{k}\right)}\right] d \eta\right]+ \\
+\theta_{1}^{(e)}\left[(\lambda+2 \mu) \int_{0}^{h} N_{i} \frac{\partial N_{1}}{\partial \eta}\left(\eta+r_{k}\right) d \eta\right]+ \\
+U_{2}^{(e)}\left[(\lambda+2 \mu) \int_{0}^{h}\left[\left(\eta+r_{k}\right) \frac{\partial N_{i}}{\partial \eta} \frac{\partial N_{2}}{\partial \eta}+\frac{N_{i} N_{2}}{\left(\eta+r_{k}\right)}\right] d \eta\right]+ \\
+\theta_{2}^{(e)}\left[(\lambda+2 \mu) \int_{0}^{h} N_{i} \frac{\partial N_{2}}{\partial \eta}\left(\eta+r_{k}\right) d \eta\right]+ \\
+\ddot{U}_{1}^{(e)}\left[\int_{0}^{h} \rho c_{1}^{2} N_{i} N_{1} d \eta+\ddot{U}_{2}^{(e)}\left[\int_{0}^{h} \rho c_{1}^{2} N_{i} N_{2} d \eta\right]=\right. \\
=\left.(\lambda+2 \mu) N_{i} \frac{\partial u}{\partial \eta}\left(\eta+r_{k}\right)\right|_{r_{k}} ^{r_{k+1}}
\end{gathered}
$$$$
\Theta_{1}^{(e)}\left[\int_{0}^{h} \frac{\partial N_{i}}{\partial \eta} \frac{\partial N_{1}}{\partial \eta}\left(\eta+r_{k}\right) d \eta\right]+
$$$$
+\Theta_{2}^{(e)}\left[\int_{0}^{h} \frac{\partial N_{i}}{\partial \eta} \frac{\partial N_{2}}{\partial \eta}\left(\eta+r_{k}\right) d \eta\right]+
$$$$
+\dot{\Theta}_{1}^{(e)}\left[\int_{0}^{h} N_{i} N_{1}\left(\eta+r_{k}\right) d \eta\right]+\dot{\Theta}_{1}^{(e)}\left[\int_{0}^{h} N_{i} N_{2}\left(\eta+r_{k}\right) d \eta\right]+
$$$$
+\ddot{\Theta}_{1}^{(e)}\left[t_{q} \int_{0}^{h} N_{i} N_{1}\left(\eta+r_{k}\right) d \eta\right]+\ddot{\Theta}_{1}^{(e)}\left[t_{q} \int_{0}^{h} N_{i} N_{2}\left(\eta+r_{k}\right) d \eta\right]+
$$$$
+\dot{U}_{1}^{(e)}\left[\xi \int_{0}^{h} N_{i}\left(\eta+r_{k}\right)\left(\frac{\partial N_{1}}{\partial \eta}+\frac{N_{1}}{\left(\eta+r_{k}\right)}\right) d \eta\right]+
$$$$
+\dot{U}_{2}^{(e)}\left[\xi \int_{0}^{h} N_{i}\left(\eta+r_{k}\right)\left(\frac{\partial N_{1}}{\partial \eta}+\frac{N_{1}}{\left(\eta+r_{k}\right)}\right) d \eta\right]+
$$$$
+\ddot{U}_{1}^{(e)}\left[t_{q} \xi \int_{0}^{h} N_{i}\left(\eta+r_{k}\right)\left(\frac{\partial N_{1}}{\partial \eta}+\frac{N_{1}}{\left(\eta+r_{k}\right)}\right) d \eta\right]+
$$$$
+\ddot{U}_{2}^{(e)}\left[t_{q} \xi \int_{0}^{h} N_{i}\left(\eta+r_{k}\right)\left(\frac{\partial N_{2}}{\partial \eta}+\frac{N_{2}}{\left(\eta+r_{k}\right)}\right) d \eta\right]=
$$$$
=\left.\frac{\partial \theta}{\partial \eta} N_{i}\left(\eta+r_{k}\right)\right|_{r_{k}} ^{r_{k+1}} \text {. }
$$

Therefore, in view of equations (27) and (28), the matrix form of finite element equation for a single element can be obtained as

$$
\begin{aligned}
& {\left[\begin{array}{ll}
{\left[R_{11}\right]} & {\left[R_{12}\right]} \\
{\left[R_{21}\right]} & {\left[R_{22}\right]}
\end{array}\right]\left[\begin{array}{c}
\ddot{U}_{1} \\
\ddot{\Theta}_{1} \\
\ddot{U}_{2} \\
\ddot{\Theta}_{2}
\end{array}\right]^{(e)}+} \\
& +\left[\begin{array}{ll}
{\left[Q_{11}\right]} & {\left[Q_{12}\right]} \\
{\left[Q_{21}\right]} & {\left[Q_{22}\right]}
\end{array}\right]\left[\begin{array}{c}
\dot{U}_{1} \\
\dot{\Theta}_{1} \\
\dot{U}_{2} \\
\dot{\Theta}_{2}
\end{array}\right]^{(e)}+ \\
& +\left[\begin{array}{ll}
{\left[P_{11}\right]} & {\left[P_{12}\right]} \\
{\left[P_{21}\right]} & {\left[P_{22}\right]}
\end{array}\right]\left[\begin{array}{c}
U_{1} \\
\Theta_{2} \\
U_{1} \\
\Theta_{2}
\end{array}\right]^{(e)}=\left[\begin{array}{l}
J_{1} \\
g_{1} \\
J_{2} \\
g_{2}
\end{array}\right]^{(e)}
\end{aligned}
$$

where

$$
\begin{aligned}
& {\left[P_{11}\right]=\left[\begin{array}{ll}
A_{11}^{11} & A_{11}^{12} \\
A_{11}^{21} & A_{11}^{22}
\end{array}\right],\left[P_{12}\right]=\left[\begin{array}{ll}
A_{12}^{11} & A_{12}^{12} \\
A_{12}^{21} & A_{12}^{22} \\
A_{12}^{11} & A_{22}^{12}
\end{array}\right]} \\
& {\left[P_{21}\right]=\left[\begin{array}{ll}
A_{21}^{11} & A_{21}^{12} \\
A_{21}^{21} & A_{21}^{22}
\end{array}\right],\left[P_{22}\right]=\left[\begin{array}{ll}
A_{22}^{11} & A_{22}^{12} \\
A_{22}^{21} & A_{22}^{22}
\end{array}\right]} \\
& {\left[Q_{11}\right]=\left[\begin{array}{cc}
\dot{A}_{11}^{11} & \dot{A}_{11}^{12} \\
\dot{A}_{11}^{21} & \dot{A}_{11}^{22}
\end{array}\right],\left[Q_{12}\right]=\left[\begin{array}{cc}
\dot{A}_{12}^{11} & \dot{A}_{12}^{12} \\
\dot{A}_{12}^{21} & \dot{A}_{12}^{22}
\end{array}\right]} \\
& {\left[Q_{21}\right]=\left[\begin{array}{cc}
\dot{A}_{21}^{11} & \dot{A}_{21}^{12} \\
\dot{A}_{21}^{21} & \dot{A}_{21}^{22}
\end{array}\right],\left[Q_{22}\right]=\left[\begin{array}{cc}
\dot{A}_{22}^{11} & \dot{A}_{22}^{12} \\
\dot{A}_{22}^{21} & \dot{A}_{22}^{22}
\end{array}\right]} \\
& {\left[R_{11}\right]=\left[\begin{array}{cc}
\ddot{A}_{11}^{11} & \ddot{A}_{11}^{12} \\
\ddot{A}_{11}^{21} & \ddot{A}_{11}^{22}
\end{array}\right],\left[R_{12}\right]=\left[\begin{array}{cc}
\ddot{A}_{12}^{11} & \ddot{A}_{12}^{12} \\
\ddot{A}_{12}^{21} & \ddot{A}_{12}^{22}
\end{array}\right]} \\
& {\left[R_{21}\right]=\left[\begin{array}{cc}
\ddot{A}_{21}^{11} & \ddot{A}_{21}^{12} \\
\ddot{A}_{21}^{21} & \ddot{A}_{21}^{22}
\end{array}\right],\left[R_{22}\right]=\left[\begin{array}{ll}
\ddot{A}_{22}^{11} & \ddot{A}_{22}^{12} \\
A_{22}^{21} & \ddot{A}_{22}^{22}
\end{array}\right]}
\end{aligned}
$$

with

$$
\begin{gathered}
A_{i j}^{11}=\left[(\lambda+2 \mu) \int_{0}^{h}\left[\left(\eta+r_{k}\right) \frac{\partial N_{i}}{\partial \eta} \frac{\partial N_{j}}{\partial \eta}+\frac{N_{i} N_{j}}{\left(\eta+r_{k}\right)}\right] d \eta\right] \\
\dot{A}_{i j}^{11}=0, \ddot{A}_{i j}^{11}=\left[\int_{0}^{h} \rho c_{1}^{2} N_{i} N_{j} d \eta\right] \\
A_{i j}^{12}=\left[(\lambda+2 \mu) \int_{0}^{h} N_{i} \frac{\partial N_{j}}{\partial \eta}\left(\eta+r_{k}\right) d \eta\right] \\
\dot{A}_{i j}^{12}=0, \ddot{A}_{i j}^{12}=0 \\
A_{i j}^{21}=0 \\
\dot{A}_{i j}^{21}=\left[\xi \int_{0}^{h} N_{i}\left(\eta+r_{k}\right)\left(\frac{\partial N_{j}}{\partial \eta}+\frac{N_{j}}{\left(\eta+r_{k}\right)}\right) d \eta\right] \\
\ddot{A}_{i j}^{21}=\left[\int_{0}^{h} t_{q} \xi\left[\frac{\partial N_{j}}{\partial \eta} N_{i}+\frac{N_{i} N_{j}}{\left(\eta+r_{k}\right)}\right]\left(\eta+r_{k}\right) d \eta\right] \\
A_{i j}^{22}=\left[\int_{0}^{h} \frac{\partial N_{i}}{\partial \eta} \frac{\partial N_{j}}{\partial \eta}\left(\eta+r_{k}\right) d \eta\right] \\
\dot{A}_{i j}^{22}=\left[\int_{0}^{h}\left(\eta+r_{k}\right) N_{i} N_{j} d \eta\right] \\
\ddot{A}_{i j}^{12}=\left[t_{q} \int_{0}^{h} N_{i} N_{j}\left(\eta+r_{k}\right) d \eta\right]
\end{gathered}
$$


Also, the load vector can be written as

$$
\left.\begin{array}{c}
J_{1}=-\left.(\lambda+2 \mu) \frac{\partial u}{\partial \eta}\left(\eta+r_{k}\right)\right|_{r_{k}} \\
J_{2}=\left.(\lambda+2 \mu) \frac{\partial u}{\partial \eta}\left(\eta+r_{k}\right)\right|_{r_{k+1}} \\
g_{1}=-\left.\left(\frac{K^{*}}{a_{0}}+\frac{\partial}{\partial t}\right) \frac{\partial u}{\partial \eta}\left(\eta+r_{k}\right)\right|_{r_{k}} \\
g_{2}=\left.\left(\frac{K^{*}}{a_{0}}+\frac{\partial}{\partial t}\right) \frac{\partial u}{\partial \eta}\left(\eta+r_{k}\right)\right|_{r_{k+1}}
\end{array}\right\} .
$$

Hence, from equations (29-32) and the boundary conditions (12-13), the global matrix of system of differential equations for the whole disk are written in the form

$$
R \ddot{\Omega}+Q \dot{\Omega}+P \Omega=f
$$

where

$$
f=\left\{\begin{array}{c}
\lambda U_{1}-(\lambda+2 \mu) \theta_{1} \\
0 \\
0 \\
\cdot \\
0
\end{array}\right\}_{(n-2)}, \Omega=\left\{\begin{array}{c}
U_{1} \\
U_{2} \\
\cdot \\
\cdot \\
\cdot \\
\Theta_{n-1} \\
\Theta_{n}
\end{array}\right\}_{(n-2)}
$$

and the matrices $R, \mathrm{Q}, P$ are global matrices corresponding to the coefficients $\left[\begin{array}{ll}{\left[R_{11}\right]} & {\left[R_{12}\right]} \\ {\left[R_{21}\right]} & {\left[R_{22}\right]}\end{array}\right],\left[\begin{array}{ll}{\left[Q_{11}\right]} & {\left[Q_{12}\right]} \\ {\left[Q_{21}\right]} & {\left[Q_{22}\right]}\end{array}\right]$ and $\left[\begin{array}{ll}{\left[P_{11}\right]} & {\left[P_{12}\right]} \\ {\left[P_{21}\right]} & {\left[P_{22}\right]}\end{array}\right]$ of element equation (29), respectively.

\section{1. Discretization in time}

Now, to obtain the solution of equation (33) we further use the finite element approach for the time domain. For this, we divide the time domain into the nodes of length $2 \Delta t$ with nodal unknowns $\Omega_{k-1}, \Omega_{k}, \Omega_{k+1}$ and the shape functions $M_{1}, M_{2}$, and $M_{3}$. The function $\Omega$ for interval $\left[t_{k-1}, t_{k+1}\right]$ can be approximated as [19]

$$
\Omega=M_{1} \Omega_{i-1}+M_{2} \Omega_{i}+M_{3} \Omega_{i+1}
$$

where the shape functions are assumed as

$M_{1}(t)=-\frac{1}{2} p(1-p), M_{2}=(1+p)(1-p), M_{3}=\frac{1}{2} p(1+p)$

and

$$
p=\frac{t-t_{k}}{t_{k+1}-t_{k}}=\frac{t-t_{k}}{\Delta t} \text { for } t_{k-1} \leq t \leq t_{k+1}
$$

so, $d p=d t / \Delta t$.

Therefore, from equations (34-36), we have

$$
\dot{\Omega}(t)=\frac{1}{\Delta t}\left[(-1 / 2+p) \Omega_{k-1}-2 p \Omega_{k}+(1 / 2+p) \Omega_{k+1}\right],
$$

$$
\ddot{\Omega}(t)=\frac{1}{(\Delta t)^{2}}\left[\Omega_{k-1}-2 \Omega_{k}+\Omega_{k+1}\right] .
$$

Similarly, the vector $f$ may be written as

$$
f=M_{1}(t) f_{k-1}+M_{2}(t) f_{k}+M_{3}(t) f_{k+1} .
$$

Now, by applying the Galerkin's approach on equation (33) and using equations ((34), (37-39)), we obtain

$$
\begin{aligned}
& \int_{-1}^{1} w\left\{R \frac{1}{(\Delta t)^{2}}\left[\Omega_{k-1}-2 \Omega_{k}+\Omega_{k+1}\right]+\right. \\
& +Q \frac{1}{\Delta t}\left[(-1 / 2+p) \Omega_{k-1}-2 p \Omega_{k}+(1 / 2+p) \Omega_{k+1}\right]+ \\
& +P\left[-\frac{1}{2} p(1-p) \Omega_{k-1}+(1+p)(1-p) \Omega_{k}+\right. \\
& \left.\left.+\frac{1}{2} p(1+p) \Omega_{k+1}\right]\right\} d p=\int_{-1}^{1} w\left[-\frac{1}{2} p(1-p) f_{k-1}+\right. \\
& \left.+(1+p)(1-p) f_{k}+\frac{1}{2} p(1+p) f_{k+1}\right] d p
\end{aligned}
$$

where, $w$ is the weight function and $f_{k-1}, f_{k}, f_{k+1}$ are the load vectors at nodal points of time element. All shape functions ( $\left.M_{1}, M_{2}, M_{3}\right)$ can be considered as weight functions.

It has been verified that $M_{3}$ shows the most favorable stability and accuracy characteristics [19]. So, here we have considered $M_{3}$ as weight function and hence from equation (40), we have

$$
F_{1} \Omega_{k+1}=F_{2} \Omega_{k}+F_{3} \Omega_{k-1}+G
$$

where

$$
\begin{gathered}
F_{1}=R \int_{-1}^{1} M_{3} d p+D \Delta t \int_{-1}^{1} M_{3}(1 / 2+p) d p+ \\
\quad+P(\Delta t)^{2} \int_{-1}^{1} \frac{1}{2} M_{3} p(1+p) d p
\end{gathered}
$$

$$
\begin{aligned}
F_{2}= & 2 R \int_{-1}^{1} M_{3} d p+2 D \Delta t \int_{-1}^{1} M_{3} p d p+ \\
& +P(\Delta t)^{2} \int_{-1}^{1} \frac{1}{2} M_{3}(1-p)(1+p) d p
\end{aligned}
$$

$$
\begin{aligned}
F_{3}=- & R \int_{-1}^{1} M_{3} d p-D \Delta t \int_{-1}^{1} M_{3}(-1 / 2+p) d p+ \\
& +P(\Delta t)^{2} \int_{-1}^{1} \frac{1}{2} M_{3} p(1+p) d p
\end{aligned}
$$




$$
\begin{aligned}
G= & -\left[\int_{-1}^{1} \frac{1}{2} M_{3} p(1-p) d p\right](\Delta t)^{2} f_{k-1}+ \\
& +\left[\int_{-1}^{1} M_{3}(1+p)(1-p) d p\right](\Delta t)^{2} f_{k}+ \\
& -\left[\int_{-1}^{1} \frac{1}{2} M_{3} p(1+p) d p\right](\Delta t)^{2} f_{k+1} .
\end{aligned}
$$

Further, by some detailed manipulations, the equations (42-45) can be reduced to

$$
\begin{gathered}
F_{1}=R+\frac{3}{2} Q \Delta t+\frac{4}{5} P(\Delta t)^{2}, \\
F_{2}=2 R+2 Q \Delta t-\frac{2}{5} P(\Delta t)^{2}, \\
F_{3}=-R-\frac{1}{2} Q \Delta t+\frac{1}{5} P(\Delta t)^{2}, \\
G=\left[-\frac{1}{5} f_{k-1}+\frac{2}{5} f_{k}+\frac{4}{5} f_{k+1}\right](\Delta t)^{2} .
\end{gathered}
$$

Hence, from the equation (41), together with equations (46-49), yields an explicit form of system of equations, and by using this explicit form, the value at $\Omega_{k+1}$ can be determined if the nodal unknowns $\Omega_{k}$ and $\Omega_{k-1}$ are known. For the first iteration at $k=1$, we first need to calculate $\Omega_{1}$. For that, we use the Crank-Nicholson method which can be given as

$$
\begin{aligned}
{\left[\frac{2}{\Delta t} R+Q+\frac{\Delta t}{2} P\right] \Omega_{1}=} & {\left[\frac{2}{\Delta t} R+Q-\frac{\Delta t}{2} P\right] \Omega_{0}+} \\
& +2 R \dot{\Omega}_{0}+\frac{\Delta t}{2}\left[f_{0}+f_{1}\right]
\end{aligned}
$$

Now, the values of $\Omega_{0}$ and $\dot{\Omega}_{0}$ are known from the initial conditions and $f_{1}, f_{2}$ are known load vectors. Therefore, all the unknowns can now be determined by using an iterative scheme given by equations (41) and (50). This completes the theoretical development of the solution method for the present problem.

It must be pointed out that the classical Newmark time integration method can be used to formulate the solution for the time domain $[17,21]$. In the Newmark method for coupled dynamical problems, a FEM is applied to obtain the time differential system of equation and then the Newmark time integration technique is applied to get the complete solution. In the present work we have also applied Newmark algorithm to obtain the solution of equation (33) and observed a perfect match in the behavior of all field variables for the Newmark method and present FE approach.

\section{NUMERICAL RESULTS}

Now, for implementation of the method we develop our computer programming code using MATLAB to solve the problem as mentioned in the previous section. We try to have the solution to the problem by computing the temperature, displacement, and stress distributions at different times inside the hollow disk of a metallic medium under consideration of initial and boundary condition. We take the reference temperature $T_{0}=293 \mathrm{~K}$. The materialistic parameters are considered for copper metal in SI unit as [23]

$$
\begin{aligned}
& \lambda=7.76 \times 10^{10} \mathrm{~kg} \mathrm{~m}^{-1} \mathrm{~s}^{-2}, \mu=3.86 \times 10^{10} \mathrm{~kg} \mathrm{~m}^{-1} \mathrm{~s}^{-2}, \\
& T_{0}=293 \mathrm{~K}, \rho=8954 \mathrm{~kg} \mathrm{~m}^{-3}, C_{E}=383.1 \mathrm{~m}^{2} \mathrm{~K}^{-1} \mathrm{~s}^{-2}, \\
& \alpha=17.8 \times 10^{-6} \mathrm{~K}^{-1}, K=8886.73 \mathrm{~kg} \mathrm{~m} \mathrm{~K}^{-1} \mathrm{~s}^{-3} .
\end{aligned}
$$

Also, the time domain is discretized in the step size of equal length $\Delta t=0.05$ and the dimensionless values of inner and outer radii of the disk are assumed to be 1 and 10 , respectively.

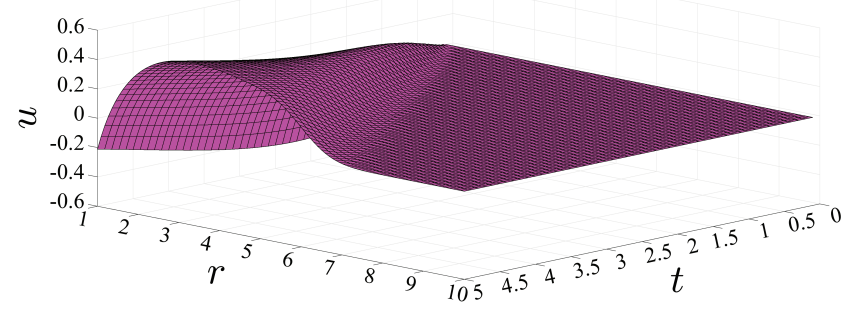

Fig. 2. Variation of $u$ vs. $r$ and $t$

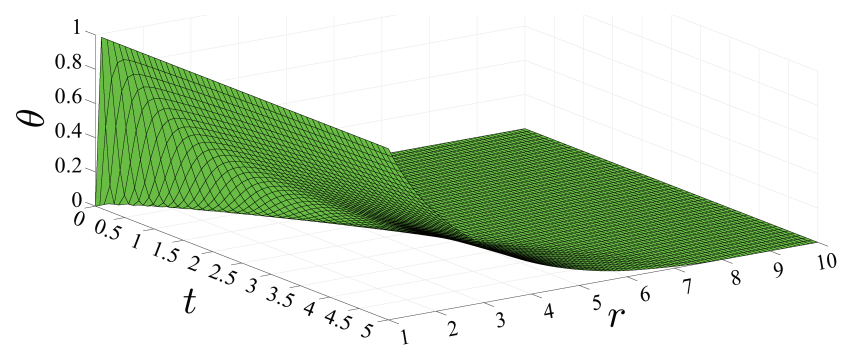

Fig. 3. Variation of $\theta$ vs. $r$ and $t$

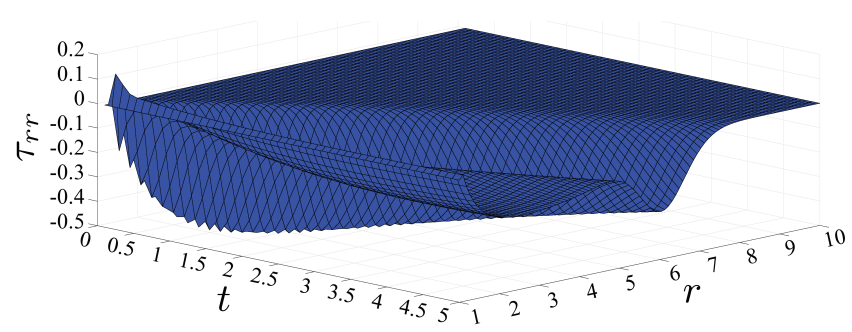

Fig. 4. Variation of $\tau_{r r}$ vs. $r$ and $t$ 


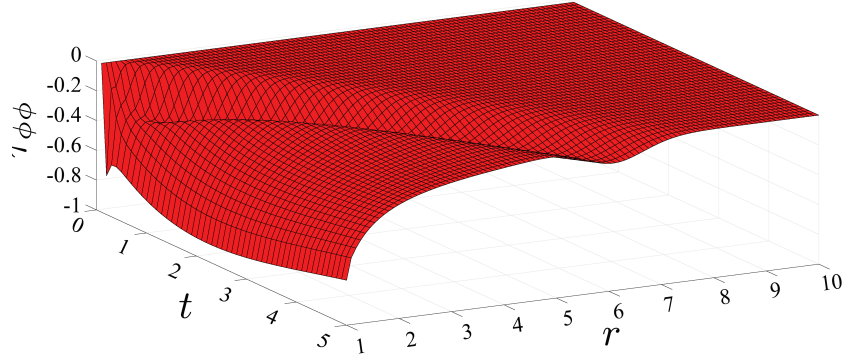

Fig. 5. Variation of $\tau_{\phi \phi}$ vs. $r$ and $t$

We take the dimensionless heat flux delay parameter $t_{q}=0.03$. In the formulation of finite element equation, we have considered the general case, but for computation, the linear Lagrangian polynomials are assumed as shape functions. For the spatial element, the hollow disk is divided into the 100 nodes of equal length along the radial direction and time domain is taken from 0 to 5 . Here, the computation is performed for the 100-time steps. The Fig. 2-5 represent the variations of the field variables (i.e. displacement, temperature, radial and circumference stresses) with respect to time and space using 3D-plots.

Fig. 2 clearly shows that at $t=0$ displacement is zero and it grows up with time. At any time displacement starts growing up from negative values at $r=1$ to attain a maximum value after some distance from the inner boundary and then decreases to zero. As time increases the maximum values of displacement increases. The region of influence increases with time.

Fig. 3 represents the variation of temperature and indicates that the temperature starts decreasing from 1 to zero value inside the disk with space. The effective domain of influence increases with the increase in time. For example, at $t=1.2$, the temperature reaches zero near $r=4.6$, while for $t=2.0$ it becomes zero at 5.2. In the Fig. 4, the radial stress $\left(\tau_{r r}\right)$ is plotted for various times and radical grids. At the inner boundary it is zero and is oscillatory in nature nearer to the inner boundary. The radial stress is compressive near the region of inner boundary and becomes tensile while reaching towards the outer boundary. Finally this field becomes zero. The region of influence increases with time. The circumferential stress $\left(\tau_{\phi \phi}\right)$ is plotted in the Fig. 5, displaying that $\tau_{\phi \phi}$ starts growing up from its minimum absolute values (at $r=1$ ) to a local maximum and then again after a local minimum it increases to zero w.r.t. radial distance. Also, with the increment of time $\tau_{\phi \phi}$ takes larger distance to reach its minimum and maximum. However, this stress is fully compressive in nature.

In order to validate the present results we have compared our solution of the problem with the corresponding solution obtained by employing the Newmark time integration method and employing the trans-finite element method which involves the Laplace transform technique for the time domain and also the numerical inversion of Laplace transform by a suitable numerical method like, Honig and Hirdes [24], Bellman et al. [25], Stehfest method [26], etc. Here, we follow the method for numerical inversion of the Laplace transform given by Bellman et al. [25]. We find that our results by the complete finite element method match with corresponding results obtained by the trans-finite element method and Newmark method. We particularly show the results of the temperature field at two different times in Fig. 6-7. The black color is used to show results for the transFEM method, the red color is used to represent the solution under the complete finite element method and the green color represents the solution under FEM with Newmark method.

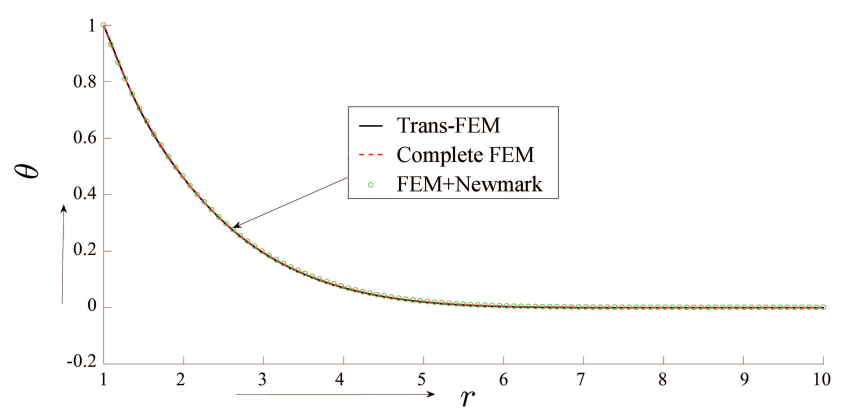

Fig. 6. Variation of $\theta$ vs. $r$, at $t=2.0$

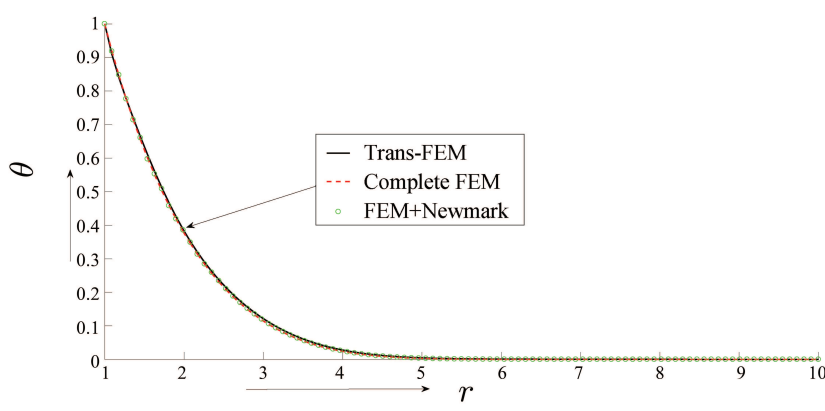

Fig. 7. Variation of $\theta$ vs. $r$, at $t=1.2$

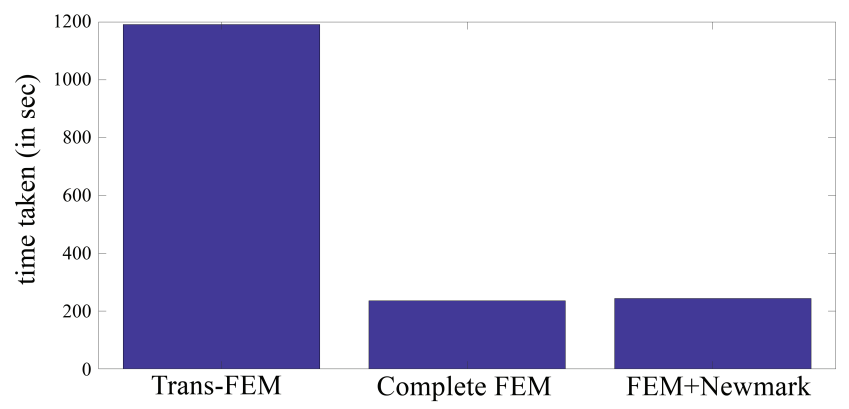

Fig. 8. Comparison of CPU time for all three methods

The similar trend of variation in the field variables and a perfect match in results validate our results and successful implementation of the complete finite element method. For showing the efficiency of the present method over the trans-FEM techniques, we have plotted a bar diagram (Fig. 8) showing the CPU time (i.e. the time taken by CPU 
to run the current MATLAB programme) for three different methods, namely, the present FEM approach, trans-FEM (involving numerical Laplace inversion) and FEM+Newmark method. It is indicated that the CPU time is much larger for trans-FEM method and approximately the same for complete FEM approach and FEM+Newmark approach. Hence, any problem on coupled thermoelasticity can be solved by using this complete finite element method as discussed in the present paper. This avoids the use of the Laplace transform method and simplifies the method for the numerical solution of the problem at any time.

\section{v. CONCLUSIONS}

We have attempted to solve a problem of linear coupled thermoelasticity based on the LS model under a complete finite element method for a homogeneous and isotropic elastic solid. In this process, the finite element equations for the present problem are derived for the space domain and time domain by discretizing in space as well as time. The validation of the results is also tested using a well-established trans-finite element method. The trans-FEMs involve numerical inversion of Laplace transform. Therefore, to apply this approach one needs to calculate the stiffness matrix for various values of Laplace parameter to obtain the solution at different time steps. In the present work we have also shown that the complete finite element is 3-4 times faster than the trans-FEM in the sense of CPU time. Therefore, it can be concluded that present finite element approach can be used as an alternative method of the FEM approach with Newmark scheme and can be more advantageous as compared to the trans-FEM method to solve the coupled thermodynamical problems.

\section{Acknowledgment}

The authors thankfully acknowledge the constructive suggestions by reviewer to improve the quality of the present paper. One of the authors (O.N. Shivay) thankfully acknowledges the full financial assistance of the JRF Fellowship (the reference no. 21/06/2015(i) EU-V, Roll no. 433492) by the University Grant Commission (UGC), India, to carry out the present work.

\section{References}

[1] M.A. Biot, Thermoelasticity and irreversible thermodynamics, Journal of Applied Physics 27, 240-253 (1956).

[2] D.S. Chandrasekharaiah, Thermoelasticity with second sound: A review, Applied Mechanics Reviews 39(3), 355376 (1986).

[3] D.S. Chandrasekharaiah, Hyperbolic thermoelasticity: A review of recent literature, Applied Mechanics Reviews 51(12) 705-729 (1998).

[4] C. Cattaneo, A form of heat conduction equation which eliminates the paradox of instantaneous propagation, Compte Rendus 247, 431-433 (1958).

[5] P. Vernotte, Les paradoxes de la theorie continue de l'equation de la chaleur, Compte Rendus 246, 3154-3155 (1958).
[6] P. Vernotte, Some possible complications in the phenomena of thermal conduction, Compte Rendus 252, 2190-2191 (1961).

[7] H.W. Lord, Y.A. Shulman, Generalized dynamical theory of thermoelasticity, Journal of the Mechanics and Physics of Solids 15(5), 299-309 (1967).

[8] A.E. Green, K.A. Lindsay, Thermoelasticity, Journal of Elasticity 2, 1-7 (1972).

[9] A.E. Green, P.M. Naghdi, A re-examination of the base postulates of thermomechanics, Proceedings: Mathematical and Physical Sciences 432, 171-194 (1991).

[10] A.E. Green, P.M. Naghdi, On undamped heat waves in an elastic solid, Journal of Thermal Stresses 15, 253-264 (1992).

[11] A.E. Green, P.M. Naghdi, Thermoelasticity without energy dissipation, Journal of Elasticity 31, 189-208 (1993).

[12] R.B. Hetnarski, J. Ignaczak, Generalized thermoelasticity, Journal of Thermal Stresses 22, 451-476 (1999).

[13] R.B. Hetnarski, M.R. Eslami, Thermal stresses: Advanced theory and applications [In:] G.M.L. Gladwell, J.R. Barber, A. Klarbring (eds), Solid mechanics and its applications, 158, Dordrecht, The Netherlands, Springer (2010).

[14] G.S. Prakash, S.S. Reddy, S.K. Das, T. Sundararajan, K.N. Seetharamu, Numerical modeling of microscale effects in conduction for different thermal boundary conditions, $\mathrm{Nu}-$ merical Heat Transfer, Part A: Applications 38, 513-532 (2000).

[15] S.C. Mishra, T.B.P. Kumar, B. Mondal, Lattice Boltzmann method applied to the solution of energy equation of a radiation and non-Fourier heat conduction problem, Numerical Heat Transfer, Part A: Applications 54(8), 798-818 (2008).

[16] B. Xu, B.Q. Li, Finite element solution of non-Fourier thermal wave problems, Numerical Heat Transfer: Part B: Fundamentals 44, 45-60 (2003).

[17] A. Bagri, M.R. Eslami, Generalized coupled thermoelasticity of functionally graded annular disk considering the LordShulman theory, Composite Structures 83, 168-179 (2008).

[18] S. Kothari, S. Mukhopadhyay, Study of a problem of functionally graded hollow disk under different thermoelasticity theories. An analysis of phase-lag effects, Computers \& Mathematics with Applications 66, 1306-1321 (2013).

[17] M.A. Rincon, B.S. Santos, J. Limaco, Numerical method, existence and uniqueness for thermoelasticity system with moving boundary, Computational \& Applied Mathematics 24(3), 439-60 (2005).

[18] I.A. Abbas, S.F. Alzahrani, A Green-Naghdi model in a 2D problem of a mode I crack in an isotropic thermoelastic plate, Physical Mesomechanics 21(2), 99-103 (2018).

[19] F.L. Stasa, Applied finite element formulation for thermal stress analysis, CBS Publishing, New York (1985).

[20] M. Balla, Formulation of coupled problems of thermoelasticity by finite elements, Periodica Polytechnica Mechanical Engineering 33(1-2), 59 (1989).

[23] H.H. Sherief, H.A. Salah, A half space problem in the theory of generalized thermoelastic diffusion, International Journal of Solids and Structures 42(15), 4484-4493 (2005).

[24] G. Honig, U. Hirdes, A method for the numerical inversion of Laplace transforms, Journal of Computational and Applied Mathematics 10, 113-132 (1984).

[25] R.E. Bellman, R.E. Kalaba, J.A. Lockett, Numerical inversion of the Laplace transform: Applications to biology, economics, engineering and physics, American Elsevier (1966).

[26] H. Stehfest, Numerical inversion of Laplace transform, Communications of the ACM 13(1), 47-49 (1970).

[21] N.M. Newmark, A method of computation for structural dynamics, J. Eng. Mech. Div. ASCE 85, 67-94 (1959). 

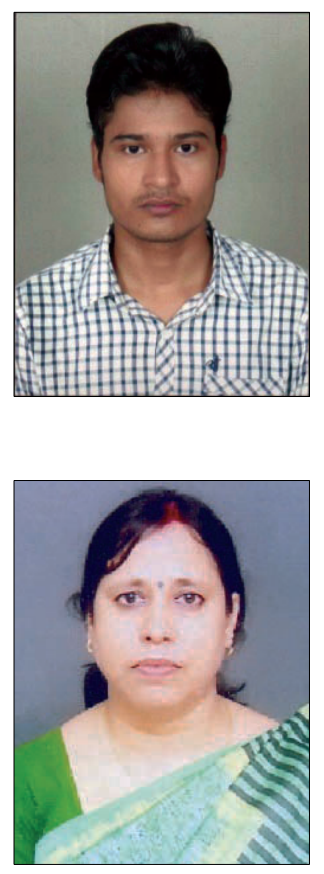

Om Namha Shivay received his Master in Science degree in Mathematics from the Banaras Hindu University, Varanasi, India, in 2015. He is doing research from the Indian Institute of Technology (BHU) in the area of Mathematical Modelling on Generalized Thermoelasticity and he has strong interest in application of computational methods for solution of coupled thermomechanical problems. He has published 3 papers in reputed journals.

Santwana Mukhopadhyay completed her PhD in Applied Mathematics at the University of Burdwan, India in 1998. Currently she is working as a Professor at the Department of Mathematical Sciences, Indian Institute of Technology (BHU), India. She has published more than 85 research papers in peer-reviewed reputed journals, and more than 20 conference presentations in her professional area of the theory of thermoelasticity and computational mathematics. She is active reviewer and editor/associate editor of various international journals. 